\title{
Is the united intelligence response, the end of speciesism and the emergence of new avatarism?
}

\author{
Susana C. Silva \\ Católica Porto Business School - Universidade Católica Portuguesa \& CEGE \\ ssilva@ucp.pt \\ Agnis Stibe \\ Metis Lab, EM Normandie Business School, Paris, France \\ INTERACT Research Unit, University of Oulu, Oulu, Finland \\ agnis@,agnisstibe.com \\ Roberta De Cicco \\ University of Chieti/Pescara \\ roberta.decicco@unich.it
}

\begin{abstract}
With the advancements in artificial intelligence (AI) research, the "algorithm aversion" concept has become evident, referring to situations in which an algorithm consistently beats human judgment, despite people's preference for interaction with humans. Moreover, another idea following this one emerged: the concept of "uncanny valley" referring to people feeling comfortable dealing with AI for solving more cognitive-oriented tasks and less prone to condescend to, share feelings with, and feel less proximity to it, for more emotional experiences.

Despite this visible aversion but apparent reliability in AI mechanisms, the truth is that AI seems to be inescapable in different fields, inclusively in the interaction with the public. Thus, it is probably time to ask ourselves if living along with these forms of intelligence is not inevitable, with full respect for each other's idiosyncrasies. And, if it is not about time either to start foreseeing the world as a place where a united intelligence response would eventually emerge and give space for a new avatarism era. This manuscript aims to start immersing in this quest.
\end{abstract}

Keywords Artificial intelligence, Speciesism, United intelligence, Human-computer symbiosis, Alternative man-machine co-evolution

\section{Introduction}

Even though some animals are known to be superior to humans in many mental or perceptual tasks and the conscience of this has increased over time (Griffin, 2013), human intelligence has been still recognized in its superiority over many other forms of intelligence on earth. That axiom seems to be gaining adepts, namely in the era of artificial intelligence (AI) advancement like the one we are living in now. Yet, we have reached a stage where AI is convincingly surpassing human capabilities in many ways. Besides, we have witnessed a trend in which AI permeates all the significant aspects of human life, including how we do business and manage organizations. AI is one of the most-discussed technology trends in academic research and practice today. It is estimated to deliver an additional global economic output of around USD 13 trillion by 2030 
(Bughin et al., 2018). The pace at which this permeability was expected to occur fifty years ago is not yet still achieved. However, it is time to act in anticipation and understand the emergence and development of this form of intelligence, which is different from humans and assumes roles that can no longer be considered inferior to humans. Despite this advancement, we are, at the same time, confronted with a stream of researchers that reinforce the superiority already mentioned and that support human speciesism (Schmitt, 2020), considering human beings to have a moral status superior to that of non-human: animals and computers.

Several studies report skepticism and ethical concerns concerning the role AI is already playing in our societies. This observation is visible in terms of data protection, privacy (switching individuals' faces in images or videos via Deepfakes generated with neural networks (Turton and Martin 2020) jobs elimination, and use of AI abilities to demonstrate its superiority over humans. This idea strengths the speciesism argument. These studies seem to endorse the so called "algorithm aversion" claiming that when an algorithm consistently beats human judgment, people prefer to bet on humans (Dietvorst, Simmons and Massey, 2015). The problem here is that humans understand how human errors happen, while computer errors often appear too stupid to be true. Computer/statistical errors look sillier somehow and therefore appear unnatural. In fact, experiments reveal that when people see algorithms making occasional mistakes, they lose confidence more quickly than when the same mistakes are made by humans (Dietvorst, Simmons and Massey, 2015).

The ground-breaking advances in the subfields of machine learning and deep learning that have been made since the early 2010s and the increasing rate at which those advances are made are visible (Thiebes, Lins and Sunyaev, 2021). Ten years after the first chatterbot (Cleverbot) to have passed the Turing Test, and to have be considered the most advanced and human-like conversational agent available, it is probably time to ask ourselves: "Are humans still managing artificial intelligence, or is that just an illusion? Is the superior intelligence looked for derived from our conception of human intelligence? Alternatively, is this a different intelligence requiring a different conception of moral?" In the following lines, we present reasoning concerning the end of this illusion.

\section{The philosophical stance}

Being the concept of intelligence already tricky to define when concerning humans, one should not expect it to be more accessible when applying it to machines. Moreover, as there are different levels and forms of intelligence in humans, how could we expect this notion to be free of doubts and rigorous if applied to an algorithm? It would not be more reasonable to realize that different forms of intelligence exist. The only possible way to dilute these would be letting both - humans and computers - understand each other's differences and follow similar ways, without assuming one to be superior to the other?

When this debate takes a philosophical stance, we have arguments pro and con considering human beings as superior to other non-human beings. At first sight, it seems reasonable to agree with Kant on human beings having both worth and dignity, while animals (or machines) do not. The Kantian philosophy produces valuable insights for the debate whether human beings are superior to others, 
with the defense of the value of autonomy and self-consciousness in maintaining that "man" prevails in the end in its superiority. However, the Kantian approach will not help those whose objective is to demonstrate that all human beings have superior status to non-human animals. This is because this approach recognises that - in some cases - some animals have superior cognitive abilities to humans (Singer, 2009), or machines. Kant can, thus, be used - to some extent - to withstand equal treatment of species. However, if we rely on the Utilitarianism idea (Bentham, 1789) - of the maximization of the good - we would need to consider the supremacy of humans over non-humans. Imagine that an AI self-driving car is faced with a terrible decision: crash into an old man on the road with little damage for the car or crash and destroy itself against the side of a mountain. The solution looks simple. The followers of the practical approach advise that we should ask ourselves: 'Can they (non-humans) suffer?', instead of 'Can they reason?' or 'Can they talk?'. As machines seem not to suffer, we could consider human supremacy. A second aspect worth mentioning regarding the debate on the differences between human vs non-human is the "consciousness" dilemma. AI scientists face the dilemma of whether they could ever create a conscious machine, or it will be a simple machine that can only follow the command to mimic the moral action (Nath and Manna, 2021). Today, only those who have a conscience can be considered agents of that particular action, and robots and AI are currently simply programmed systems capable enough to accomplish the task assigned to it, whether with a positive or with a negative outcome. But, what happens when agents will be competent enough to perform any moral action?

We tend to agree with Singer (2009) that human beings must maintain the standard ethical view that every human life is of equal value. This idea is precisely the argument used by the Pope John Paul II, even though we understand that not being precisely what reality shows, and even though we understand that is what indeed throws machines to a secondary level. Philosophy has led to the apparent differences between human beings (races do not have inherent physical or biological meaning) and humans and non-humans (Korsgaard, 2011). In this debate, humans are provided different opportunities to enjoy the life to come, which is an instrumental argument to withstand the supremacy of humans over machines. However, this would also allow us to project ourselves to a future situation: a turning point - whatever this would be -, where AI developments can be considerably different from what we have so far.

In a future that can be nearer than one could expect, we can have a situation in which selfconsciousness and inherent suffering may become characteristics of superior forms of intelligence yet to come. In such a scenario, Whitby's (2008) question arises: is it acceptable to treat artefacts - particularly human-like artefacts - in ways that we would consider it morally unacceptable to treat humans? There is already research on the defense of robot's rights (Robertson, 2014), especially - but not only - in what concerns the moral and sexual harassment that some have been experiencing (Curry \& Rieser, 2018), and that deserves further investigation. The case of Bia, from Bradesco, and how this chatbot was programmed to respond to provocations, especially the sexualcontented ones (Silva, 2021), paves the way for future research of robots' rights and their possible ability to create self-consciousness and profess feelings.

\section{A possible response}

To properly assess the question that gives tile to this research, we need to project ourselves in the future. We argue that future intelligence should not be seen anymore as embedded in agents or encapsulated within bodies, but rather considered information, out of form, made available to 
everyone, as an 'undepletable' source that anyone can access, expand, and improve, for individual and group's access. This means that people and machines, as form-shaped agents, can learn to develop tools and technologies to master better lives and establish more successful businesses. These tools are available, either produced by machines or by humans. Furthermore, intelligence can be developed further by both, in a co-creative (the time is not far away when creative content will be made by robots, e.g. Nicolic and Young, 2010), co-evolutive and symbiotic process. One need only think of Neuralink Corporation, the American company founded in 2016 by Elon Musk, which aims to create an implantable Brain-Machine Interface (BMI) and enhance biological human brain capacity. This blended knowledge, which is now occupying a space, will tend to be de-materialized and embedded in one's minds in a progressively natural way. To get there, we must develop our social interaction abilities with machines. For example, space agencies like NASA and ISRO are replacing human astronauts with humanoid robots for their next space expeditions, and computer scientists must work side-by-side with people and machines from multiple backgrounds. AI scientists are already looking forward to uploading the human brain in a supercomputer. In the science fiction thriller "Transcendence", scientists have already uploaded people's consciousness into the quantum computer. Even though this still falls in the bucket of post-modern science, such an approach would represent a significant shift from today's humancentered Artificial Intelligence model, as Schmidt (2020) proposed a futuristic Man-Machine Unified Intelligence Model.

The ability of each human to absorb this intelligence will depend on their innate ability to learn things. Still, it is not deterministic, as more and more, neuralink.com possibilities will be at the disposal of everyone, thus enabling augmenting the intelligence of humans. In the same vein, as this source of information becomes universally accessible, machines will infuse and breed and inbreed from it, taking their possibilities to the next level. Even if far in the present, these scenarios are being anticipated with consequences over different areas of our life, from medicine to agriculture, from geophysics to mining, passing by business. In business, individuals (both employees and consumers) and machines can establish a fruitful synergy towards achieving organizational hyper-performance. The ultimate example of this is the paradigm shift from the shopping-then-shipping to the shipping-then-shopping (Agrawal et al., 2018).

\section{What consequences on business?}

The uncanny valley paradox is caused by people ascribing machines different characteristics than humans and feeling less prone to condescend to, share feelings with them, and feel less proximity to them. At the same time, as these are not humans, people interacting with them may feel more comfortable trusting them for more-oriented tasks. This psychological comfort with AI used for more cognitive-oriented tasks comes from the human tendency to associate agency with human capacities for thought, and associate experience with human capacities for feelings and emotions. Machines cannot have conscious emotional experiences, so people perceive them as having capacities for cognition but not emotion (Bakpayev et al., 2021). Managers are likely to appreciate this psychological comfort, though, as explore AI further especially in an era where the amounts of data available are tremendous and the limitations to handle it all, huge.

Corporate management increasingly relies on intelligent systems that can bring quick and meaningful insights, especially about the relevant key performance indicators and data related to consumer behaviour. Even though consumers may display "uncanny valley paradox" (or 
"algorithm aversion" feeling) (De Cicco et al, 2020), such real-time thoughts are significant contributions to any required executive decision-making process. Since the advent of digitalization, new organizations are continuously shifting more weight onto technology-supported managerial practices. One can say that what is called management AI today are not more than complex advanced statistical tools, such as neural networks, which can exploit large amounts of data and have been given appealing names. Therefore, it is not surprising that they are well accepted by managers. They are a tool like excel was before them. Many companies already use sophisticated solutions for instant data collection and analysis, which means that machines gain access to many data that can no longer be in complete control of corporate leadership. So, this also means that computer networks contain huge quantities of data. This data may inclusively derive from a new avatarism movement (Quinby, 2014). New avatarism can be seen as a new form of liberalism, in which individuals and machine co-exist under the right to have their forms protected. This protection can be, inclusively, provided by augmented reality and pseudonymously uncovered. The true identities of the entities would be reserved only for relations with authorities. Again, this places the cornerstone role on how to best blend the two sources of intelligence mentioned before, and calls for a united intelligence response. If people and machines are considered separate entities and allowed to deal differently with the information available, synergies are less likely to occur in a separate and non-complementarily way. A win-win situation would essentially depend on how a successful blend of human and AI can be achieved in many contexts, as well as in management.

As many other devices in the past, AI technology is helping humans dealing with their biological limitations (Nath and Manna, 2021). Nevertheless, its sphere of influence is not limited to biology and AI improvements are reflected in many fields. Humans use their intelligence to build an AI that can accelerate desired improvements towards organizational hyper-performance. Imitating or emulating human intelligence is important for AI improvements. But, it is not going to be through this mechanism that a computer will be able to do things that the human brain is still unable to do. A more sophisticated and intricate mechanism is necessary in the journey for this strong form of $\mathrm{AI}$ and consequent hyper-performance. There is one serious difficulty on the journey to get to it: our insufficient understanding about how exactly human intelligence emerges from the neural activity in brains. Until this issue is solved, we are generally limited to producing only narrow or weak forms of AI that can only do a specific task or activity better, faster, more efficiently than humans. To put it simply, managers continue relying primarily on human intelligence to drive all the essential decision-making processes in organizations as long as the general AI has not been successfully developed and appropriated to assist with executive problem-solving needs. Once that happens, however, we will be exposed to the questions: "What kind of profound transformations will be inevitable for organizational management? Is resistance and fear from AI inevitable? What to do to guide AI for the good of Humanity?"

\section{A united intelligence}

Human nature has always been reluctant to accept new ideas and changes easily. That applies to managers, employees and consumers as well. Some leadership teams will critically and skeptically look at the decisions made and suggested by general AI. At the same time, the workforce will question if decisions were a corporate decision made by the human or artificial part of their management? There is an apparent bias towards those made by humans (speciesism) and some apparent preference for the efficiency ascertain to those determined by machines. Humans quite 
often are creating unnecessary contradictions. Similarly, it will happen at any later stage of AI, introducing new paradigms in organizational culture. Many fundamental preparatory steps can be made to avoid future resistance and fear from AI, even if this will only reflect in the next 70 years.

If most current $\mathrm{AI}$ is considered narrow (or weak) and we are still heading a more bread and strong one - which some claim non-existent yet - the future is still ahead and can be anticipated, so it will be better to be anticipated right away. In the future, not just the so-called super-intelligence will be created, representing triggering questions and inclusively fear, but also, and above all, a black intelligence can emerge. A superior intelligence can produce the same consequences as humans: used for good and the bad. So, in the same vein, individuals seem to have a dark side and a thirst for superiority fed by power. Also, computers are entitled to that analogy, which, after all, gets them - again - closer to humans than one may think. After all, many AI scientists and philosophers such as Nick Bostrom, Elon Musk, and Stephen Hawking have already predicted that the AI systems could cause global human extinction if not managed properly (Nath and Manna, 2021). So, as we distinguish ourselves from machines, we tend to witness them displaying attitudes and behaviours like ours, which substitutes the uncanny valley paradox with the "Like Father, Like Son" one. If we can speak about a form of speciesism of humans over machines, we can also expect this to happen - in the future - of a form of speciesism of machines over humans.

\section{Conclusion}

So, how to solve this "double sword speciesism"? There is only one way to eliminate these idiosyncrasies in the future - entangle them so much that there will be no distinction between human and artificial intelligence, but rather a united intelligence. This united intelligence will push us to abandon the idea of the superior value of humans, replacing that with a more graduated view in which moral status depends on some aspects of cognitive ability, and that graduated view is applied both to humans and non-humans (Stinger, 2009).

Thus, the best way for mitigating the drawbacks of our human nature is to face and accept them. If we are still worried about any dangerous future possibility related to AI, maybe we should embrace a transhumanistic/posthumanistic attitude and abandon our anthropocentric approach (Nath and Manna, 2021), and consider that we can evolve together and become one united intelligence: an intelligence that co-exists, co-creates and co-evolves by unifying the best of both, while successfully eliminating the existing detriments as much as possible: a man-machine symbiosis that profoundly enhances managerial practices in organizations, as well as makes our human existence more enjoyable in general, and computers existence too. So, instead of one beating the other, both forms of intelligence would intertwin, for their good and the good of the world as we know it. Speciesism will get to an end when online, and this consequent united intelligence pushes our lives and boundaries to a blurring line separating real and virtual spaces (Jordan, 2009).

\section{References:}

Agrawal, A., Gans, J., \& Goldfarb, A. (2018). Artificial Intelligence in the Boardroom. The Corporate Board, 16-20. 
Bakpayev, M., Baek, T. H., van Esch, P., \& Yoon, S. (2020). Programmatic creative: AI can think but it cannot feel.Australasian Marketing Journal, j-ausmj.2020.04.002. doi.org/10.1016/j.ausmj.2020.04.002

Bentham, J. (1789). Introduction to the Principles of Morals and Legislation, in L'homme et lánimal. Dimensions éthiques de leus relations. Ch. 17, translated by Alberto Bondolfi, For a summary of this work: www. Angelfire. com/md2/timewarp/Bentham. HTML.

Bughin, J., Seong, J., Manyika, J., Chui, M., \& Joshi, R. (2018). Notes from the AI frontier: Modeling the impact of AI on the world economy. McKinsey Global Institute, Brussels, San Francisco, Shanghai, Stockholm.

Curry, A. C., \& Rieser, V. (2018, June). \# MeToo Alexa: How conversational systems respond to sexual harassment. Proceedings of the second ACL workshop on ethics in natural language processing (pp. 7-14).

De Cicco, R., Silva, S. and Alparone. F. (2020). Millennials' attitude toward chatbots: an experimental study in a social relationship perspective. International Journal of Retail and Distribution Management, 48(11): 1213-1233.

Dietvorst, B. J., Simmons, J. P., \& Massey, C. (2015). Algorithm aversion: People erroneously avoid algorithms after seeing them err. Journal of Experimental Psychology: General, 144(1), 114.

Griffin, D. R. (2013). Animal Minds: Beyond cognition to consciousness. University of Chicago Press.

Jordan, B. (2009). Blurring boundaries: The" real" and the" virtual" in hybrid spaces. Human Organization, 181-193.

Korsgaard, C. M. (2011). Interacting with animals: A Kantian account. Oxford Handbooks Online.

Nath, R., \& Manna, R. (2021). From posthumanism to ethics of artificial intelligence. AI \& Society, $1-12$.

Nikolic, P. K., \& Yang, H. (2020). Artificial intelligence clone generated content toward robot creativity and machine mindfulness. Mobile Networks and Applications, 25(4), 1504-1513.

Quinby, L. (2014). Eccentric Citizens: Subjectivity and Citizenship in the Technomillennium. In Gender and Apocalyptic Desire (pp. 179-193). Routledge.

Robertson, J. (2014). Human rights vs robot rights: Forecasts from Japan. Critical Asian Studies, 46(4), 571-598.

Schmidt, A. (2020). Interactive human-centred Artificial Intelligence: A Definition and Research Challenges. In Proceedings of the International Conference on Advanced Visual Interfaces (pp. 1-4).

Schmitt, B. (2020). Speciesism: an obstacle to AI and robot adoption. Marketing Letters, 31(1), 36.

Silva, S. (2021), "Quando o assédio também atinge os chatbots", om Jornal Económico oline, 27/09//2021. Available at Quando o assédio também atinge os 'chatbots' - O Jornal Económico (sapo.pt)

Singer, (2009). Speciesism and moral status, in guest editors Kittay, E. F. and Carlson, L. Cognitive disability and its challenge to moral philosophy, John Wiley \& Sons.

Thiebes, S., Lins, S., \& Sunyaev, A. (2021). Trustworthy artificial intelligence. Electronic Markets, 31(2), 447-464.

Turton, W., \& Martin, A. (2020). How Deepfakes Make Disinformation More Real Than Ever.

Whitby, B. (2008). Sometimes it's hard to be a robot: A call for action on the ethics of abusing artificial agents. Interacting with Computers, 20(3), 326-333. 
